
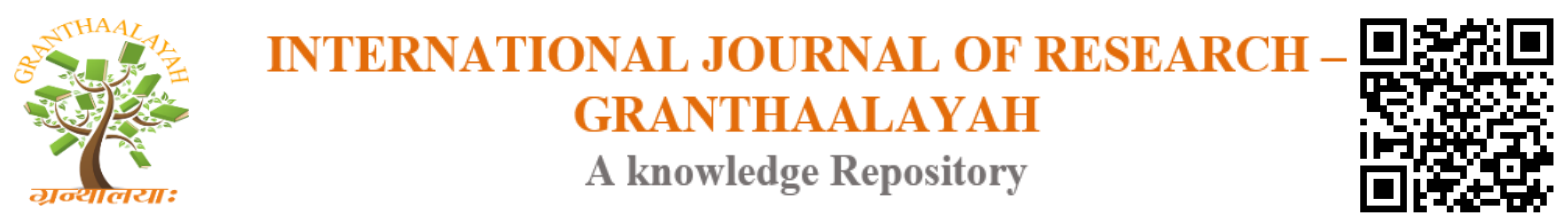

Science

\title{
ANALYSIS OF ULTRAHSORT LASER PULSES PROPAGATION IN MEDIUM: LITHARGE GLASS SF56A
}

\author{
Khelladi Mounir *1 \\ ${ }^{* 1}$ Abou-bekr Belkaid Univeristy of the Tlemcen Algeria
}

\begin{abstract}
Propagation of an ultra-short lasers pulses in a linear optical medium consisting of free space, dispersive media. However, analytical methods have the limitations of not being able to handle arbitrary pulse profiles. Also, closed form solutions are often obtained after certain levels of approximations. This has motive a few studies based on the use of numerical simulation techniques in the analysis of pulse propagation. In view of the recent advance in ultra-short pulse propagation, a strong need is felt for developing a numerical formalism capable of performing such a complete analysis of the issues involved in pulse propagation.

This allows us to analyze the pulse in the time-frequency domain at any arbitrary plane. With this tool, we investigate the spectral and temporal evolution of ultra-short pulses at any arbitrary propagation distance.
\end{abstract}

Keywords: Ultrashort Pulses; Medium Index; Femtosecond; Propagation; Dispersion; Wavelet.

Cite This Article: Khelladi Mounir. (2019). “ANALYSIS OF ULTRAHSORT LASER PULSES PROPAGATION IN MEDIUM: LITHARGE GLASS SF56A.” International Journal of Research - Granthaalayah, 7(2), 58-67. https://doi.org/10.29121/granthaalayah.v7.i2.2019.994.

\section{Introduction}

Recent developments in short pulse laser technology have led to significant advances in our fundamental understanding of ultrafast phenomena in physics, chemistry and biology, as well as stimulating the development of applications in fields as diverse as optical communications, biomedical imaging and femtosecond micromachining [1].

\section{Ultrashort Laser Pulses Generations}

The central aim of this section is to give a concise introduction to nonlinear optics and to provide basic information about the most-widely used tunable femtosecond laser sources, in particular tunable Ti:sapphire oscillators and Ti:sapphire amplifiers or optical parametric amplifiers. 


\subsection{Gaussian pulse}

In this subsection we would like to consider an example of Gaussian pulse, which is most commonly used in ultrashort laser pulse characteristics. The pulse is linearly chirped an represented by

$$
A(t)=A_{0} \exp \left(\frac{-(1+i \alpha) t^{2}}{\tau_{g}^{2}}\right) \quad \text { with } \quad \Delta \tau_{p}=\sqrt{2 \ln 2 \tau_{g}}
$$

Pulse is down-chirped for a positive chirp parameter $\alpha$, for negative is upchirped and when $\alpha=0$ then the pulse is unchirped. The spectral instensity can be derived by taking the Fourier-transform of eq.1, it also has the Gaussian shape [2].

\section{Propagation of A Light Pulse in Transparent Medium}

When an electromagnetic wave interacts with the bound electrons of a dielectric medium the medium response in general depends on the optical frequency of the wave. This property is referred to as chromatic dispersion. It manifests itself through frequency dependence of the refractive index of the medium. The frequency Fourier transform of a Gaussian pulse has already been given as

$$
E(w)=\exp \left(\frac{-\left(w-w_{0}\right)^{2}}{4 . \Gamma}\right)
$$

An ultrashort Fourier limited pulse has a broad spectrum and no chirp; when it propagates a distance through a transparent medium, the medium introduces a dispersion to the pulse inducing an increase in the pulse duration. To investigate and determine the dispersion, we assume a Gaussian shape for the pulse. The electric field of the pulse is given as Eq.2

After the pulse has propagated a distance $\mathrm{z}$, its spectrum is modified to

$$
E(w, z)=E(w) \exp [ \pm i k(w) z], \quad k(w)=\frac{n(w) \cdot w}{c}
$$

where $\mathrm{k}(\mathrm{w})$ is now a frequency-dependent propagation factor. In order to allow for a partial analytical calculation of the propagation effects, the propagation factor is rewritten using a Taylor expansion as a function of the angular frequency, assuming that $\Delta w \ll w_{0}$ (this condition is only weakly true for the shortest pulses). Applying the Taylor expansion to eq.4, the pulse spectrum becomes.

$$
\begin{gathered}
k(w)=k\left(w_{0}\right)+k^{\prime}\left(w-w_{0}\right)+\frac{1}{2} k^{\prime \prime}\left(w-w_{0}\right)^{2}+\cdots \\
\text { where } k^{\prime}=\left(\frac{d k(w)}{d w}\right)_{w_{0}} \text { and } k^{\prime \prime}=\left(\frac{d^{2} k(w)}{d w^{2}}\right)_{w_{0}} \\
E(w, z)=\exp \left[-i k\left(w_{0}\right) z-i k^{\prime} z\left(w-w_{0}\right)-\left(\frac{1}{4 \Gamma}+\frac{i}{2} k^{\prime \prime}\right)\left(w-w_{0}\right)^{2}\right] .
\end{gathered}
$$


The time evolution of the electric field in the pulse is then derived from the calculation of the inverse Fourier transform of eq.6,

so that

$$
e(t, z)=\int_{-\infty}^{+\infty} E(w, z) \cdot e^{-i w t} d w
$$

$$
e(t, z)=\sqrt{\frac{\Gamma(z)}{\pi}} \cdot \exp \left[i w_{0}\left(t-\frac{z}{V_{\emptyset}\left(w_{0}\right)}\right)\right] \times \exp \left[-\Gamma(z)\left(t-\frac{z}{V_{g}\left(w_{0}\right)}\right)^{2}\right]
$$

Where $\quad V_{\emptyset}\left(w_{0}\right)=\left(\frac{w}{k}\right)_{w_{0}}, \quad V_{g}\left(w_{0}\right)=\left(\frac{d w}{d k}\right)_{w_{0}}, 1 /\left(\Gamma(z)=1 / \Gamma+2 i k^{\prime \prime} z\right.$.

In the first exponential term of eq.7, it can be observed that the phase of the central frequency $w_{0}$ is delayed by an amount $\frac{Z}{V_{\varnothing}}$ after propagation over a distance $z$. The second term in eq. 40 shows that, after propagation over a distance $\mathrm{z}$, the pulse keeps a Gaussian envelope. This envelope is delayed by an amount $z / V_{g}, V_{g}$ being the group velocity. The second term in eq.7 also shows that the pulse envelope is distorted during its propagation because its form factor $\Gamma(z)$, defined as [2,3]

$$
1 /\left(\Gamma(z)=1 / \Gamma+2 i k^{\prime \prime} z\right.
$$

Depends on the angular frequency $w$ through $k^{\prime \prime}(w)$,

$$
k^{\prime \prime}=\left(d^{2} k / d w^{2}\right)_{w_{0}}=\frac{d}{d w}\left(\frac{1}{V_{g}}\right)_{w_{0}} .
$$

This term is called the "Group Velocity Dispersion". The temporal width of the pulse at point z:

$$
\Delta \tau_{z}=\Delta \tau_{0} \sqrt{1+4 .\left(\Gamma \cdot k^{\prime \prime} z\right)^{2}} .
$$

with $\quad k^{\prime \prime}=\frac{\lambda^{3}}{2 \cdot \pi \cdot c^{2}} \frac{d^{2} n}{d \lambda^{2}} \quad \Gamma=\frac{2 \log 2}{\Delta_{0}^{2}}$,

\subsection{Application in Litharge Index SF56-A Medium}

In optical materials, the refractive index is frequency dependent. This dependence can be calculated for a given material using a Sellmeier equation, typically of the form

$$
n^{2}(w)=1+\sum_{i=1}^{m} \frac{B_{i} w_{i}^{2}}{w_{i}^{2}-w^{2}}
$$

- High nonlinear index coefficient $\left(22-286.10^{-20}\left[\mathrm{~m}^{2} / \mathrm{W}\right]\right.$

- Wide transmission range $(0.4 \mu m \sim 3 \mu m)$

- Transition temperature $\left(\sim 700^{\circ} \mathrm{C}\right)$ and melting temperature $\left(\sim 1100^{\circ} \mathrm{C}\right)$

- Poor electrical conductivity

- Highest atomic number of all stable elements 
The index of litharge SF56-A is given by the following expression (11):

Table 1: Parameters for litharge SF56A Glasses

\begin{tabular}{|c|l|l|l|}
\hline $\boldsymbol{B}_{\boldsymbol{i}}$ & 1.70579259 & 0.0344223052 & 1.09601828 \\
\hline $\boldsymbol{\lambda}_{\boldsymbol{i}}(\boldsymbol{\mu} \boldsymbol{m})$ & 0.0133874699 & 0.0579561608 & 121.616024 \\
\hline
\end{tabular}

where $w_{i}$ is the frequency of resonance and $B_{i}$ is the amplitude of resonance. In the case of optical fibers, the parameters $w_{i}$ and $B_{i}$ are obtained experimentally by fitting the measured dispersion curves to eq.11 with $m=3$ and depend on the core constituents [4].

\subsection{Parameter of Dispersion}

An ultrashort Fourier limited pulse has a broad spectrum and no chirp; when it propagates a distance through a transparent medium, the medium introduces dispersion to the pulse inducing an increase in the pulse duration. We consider dispersions of orders two. The pulse broadens on propagation as a result of group velocity dispersion (GVD).

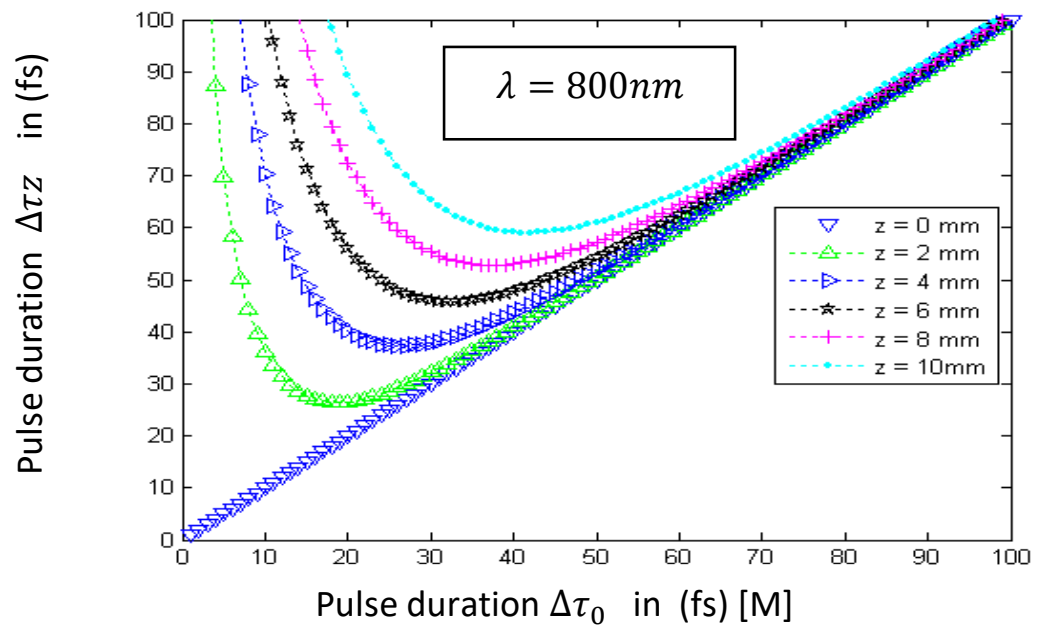

Figure 1: Temporal broadening of the transform- limited pulse for different values of the propagation distance $\mathrm{z}$.

In summary, the propagation of a short optical pulse through transparent medium results in a delay of the pulse, a duration broadening and a frequency chirp.

\subsection{Group Velocity Dispersion}

The Group Velocity Dispersion (GVD) is defined as the propagation of different frequency components at different speeds through a dispersive medium. This is due to the wavelengthdependent index of refraction of the dispersive material. GVD causes variation in the temporal profile of the laser pulse, while the spectrum remains unaltered. To the first place we limited only to the order two of the Taylor expansion of the phase. It is noticed that the analysis of Fourier remains valid only for durations of pulse which are higher than $\approx 60 \mathrm{fs}$. 
In addition, media we consider all higher order dispersion, which completely describes the physical processes involved in ultrashort dispersive pulse dynamics. The pulse broadens in time and becomes asymmetric [5]

$$
\begin{gathered}
\varphi(w)=\varphi\left(w_{0}\right)+\left(w-w_{0}\right) \frac{d \varphi}{d w}\left|+\frac{1}{2 !}\left(w-w_{0}\right)^{2} \frac{d^{2} \varphi}{d w^{2}}\right|_{w=w_{0}}+ \\
+\left.\frac{1}{n !}\left(w-w_{0}\right)^{n} \frac{d^{n} \varphi}{d w^{n}}\right|_{w=\Omega}+\theta(w){ }_{w=w_{0}} \\
\varphi(\lambda)=\frac{2 \pi}{\lambda} n(\lambda) z \\
\frac{d \lambda}{d w}=-\frac{\lambda^{2}}{2 \pi c} \\
\frac{d \varphi}{d w}=-\frac{z}{c}\left[\frac{d n}{d \lambda}-n\right] \\
\frac{d^{2} \varphi}{d w^{2}}=+\frac{\lambda^{3}}{4 \pi^{3} c^{2}} \frac{d^{2} n}{d \lambda^{2}} z \\
\frac{d^{3} \varphi}{d w^{3}}=-\frac{\lambda^{4}}{4 \pi^{2} c^{3}}\left[3 \frac{d^{2} n}{d \lambda^{2}}+\lambda \frac{d^{3} n}{d \lambda^{3}}\right] Z \\
\frac{d^{4} \varphi}{d w^{4}}=+\frac{\lambda^{5}}{8 \pi^{3} c^{4}}\left[12 \frac{d^{2} n}{d \lambda^{2}}+8 \lambda \frac{d^{3} n}{d \lambda^{3}}+\lambda^{2} \frac{d^{4} n}{d \lambda^{4}}\right] Z \\
\frac{d^{5} \varphi}{d w^{5}}=-\frac{d^{2}}{16 \pi^{4} c^{5}}\left[60 \frac{d^{2} n}{d \lambda^{2}}+60 \lambda \frac{d^{3} n}{d \lambda^{3}}+15 \lambda^{2} \frac{d^{4} n}{d \lambda^{4}}+\lambda^{3} \frac{d^{5} n}{d \lambda^{5}}\right] Z \\
\frac{\lambda^{7}}{32 \pi^{5} c^{6}}\left[360 \frac{d^{2} n}{d \lambda^{2}}+480 \lambda \frac{d^{3} n}{d \lambda^{3}}+180 \lambda^{2} \frac{d^{4} n}{d \lambda^{4}}+24 \lambda^{3} \frac{d^{5} n}{d \lambda^{5}}+\lambda^{4} \frac{d^{6} n}{d \lambda^{6}}\right] Z \\
\frac{d^{6} \varphi}{d w^{6}}=+
\end{gathered}
$$

It seems to me that we can write $\varphi^{(i)}=\frac{d^{i}}{d w^{i}}$ as a recurrence, giving $\varphi^{(i)}$ based on derivatives of order $i$, the index of refraction. Matrix form, we can write

$$
\left[\begin{array}{l}
\phi^{(2)} \\
\emptyset^{(3)} \\
\emptyset^{(4)} \\
\emptyset^{(5)} \\
\emptyset^{(6)}
\end{array}\right]=(-1)^{n} 2 . \pi \cdot z\left[\frac{\lambda}{2 . \pi \cdot c}\right]^{n}\left[\begin{array}{lllll}
1 & 0 & 0 & 0 & 0 \\
3 & 1 & 0 & 0 & 0 \\
12 & 8 & 1 & 0 & 0 \\
60 & 60 & 15 & 1 & 0 \\
360 & 480 & 180 & 24 & 1
\end{array}\right]
$$

The various terms of the Taylor expansion to order $n$ can be written in the shape of a matrix [A], which's we can express various terms A ij.

$$
\begin{array}{r}
\emptyset(w)=\varnothing\left(w_{0}\right)+\left(w-w_{0}\right) \emptyset^{(1)}+\left.\sum_{i=2}^{p} \frac{1}{i !}\left(w-w_{0}\right)^{i} \emptyset^{(i)}\right|_{w=w_{0}}+\theta(w) . \\
\emptyset^{(p)}=(-1)^{p} .2 \pi . z\left[\frac{\lambda}{2 . \pi . c}\right]^{p} \sum_{j=2}^{p} \lambda^{j-1} A(p-1, j-
\end{array}
$$

1) $n^{(j)}$ with $\quad p>2$ 

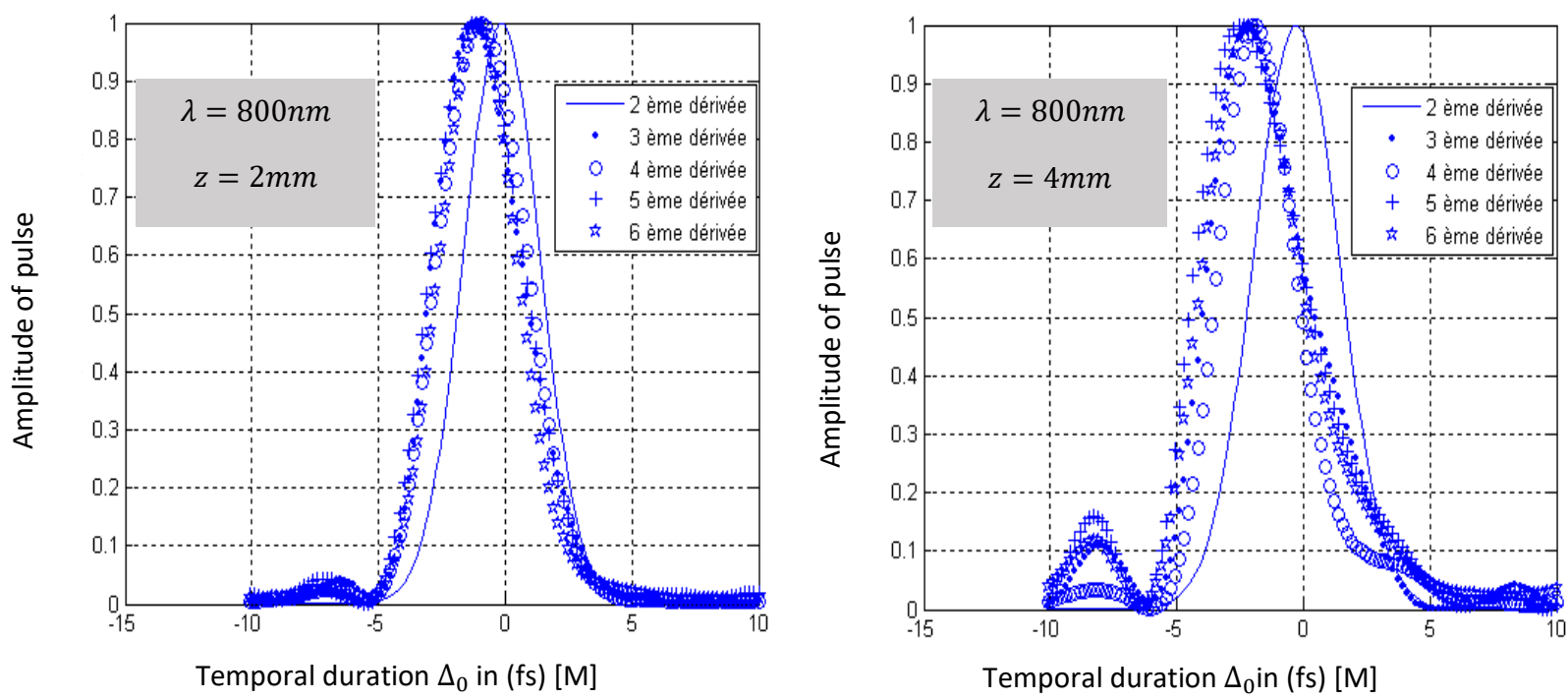

Figure 2: (a) the pulse broadens on propagation as a result of group velocity dispersion (GVD) (b) The pulse shape is no longer Gaussian and it becomes asymmetric due to higher order dispersions

Analytically observed propagation affects such as spectral shift, pulse broadening and asymmetry in dispersive media can be easily brought out in the simulation using formalism presented here. In addition, such studies can be extended to pulses of arbitrary temporal shape without any further algorithmic complexity by numerical simulation. Higher order dispersion effects can be handled easily in the numerical simulation unlike in the case of analytical calculation [8]

\section{Time-Frequency Decomposition}

\subsection{Wavelet Theory}

The wavelets are very particular elementary functions, these are the shortest vibrations and most elementary that one can consider. One can say that the wavelet east carries out a zooming on any interesting phenomenon of the signal which place on a small scale in the vicinity of the point considered [7].

\subsection{Wavelet Techniques}

Starting with a signal $e(t)$, in plane $z=0$, we define wavelet centered at $\Omega$ by

$\theta(\Omega)=E(w) \cdot \exp \left[-\frac{(w-\Omega)^{2}}{4 \gamma}\right], \quad$ with $\quad E(w)=\frac{E_{0}}{2 \cdot \pi} \sqrt{\frac{\pi}{\Gamma}} \exp \left[\frac{\left(w-w_{0}\right)^{2}}{4 \cdot \Gamma}\right]$ 


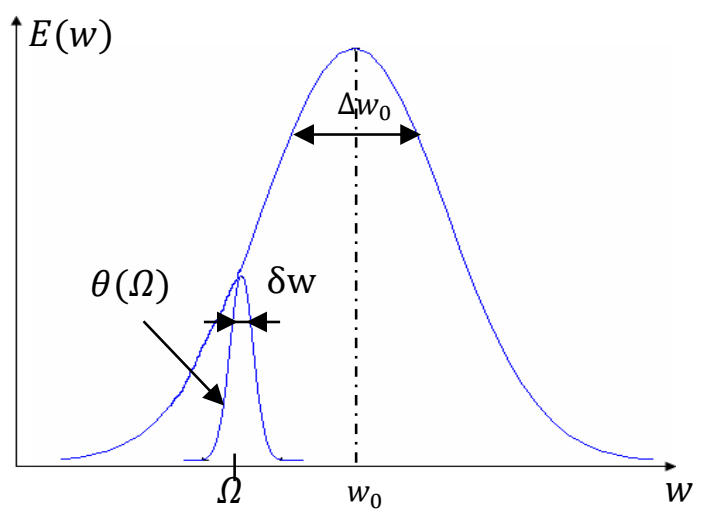

Figure 3: Gaussian envelope decomposed on a number of wavelet we calculates the electric field associated with the wavelet $\theta(\Omega, z=0)$.

$$
\begin{gathered}
\theta(t, z=0)=\operatorname{TF}\{\theta(\Omega, z=0)\} \\
\theta(t, z=0)=E_{0} \sqrt{\frac{\gamma}{\gamma+\Gamma}} \cdot \exp \left[\frac{-\left(w_{0}-\Omega\right)^{2}}{4(\gamma+\Gamma)}\right] \cdot \exp \left[-\frac{\gamma \Gamma}{\gamma+\Gamma} t^{2}\right] \cdot \exp \left[j \frac{\gamma w_{0}+\Gamma \Omega}{\gamma+\Gamma} t\right]
\end{gathered}
$$

In time, the pulse is also Gaussian, of parameter $\frac{\gamma \Gamma}{\gamma+\Gamma}$.

The maximum of amplitude of the wavelet $\theta(t, z=0)$ vary with $\Omega$, center frequency of analysis on Gaussian of parameter $\gamma+\Gamma$.

The signal propagates in the positive $z$ direction in a linear dispersive and transparent medium, which fills the half space $z>0$ and whose refractive index is $n(w)$. After propagation, the wavelet $\theta(\Omega, x)$ may be written as

$$
\theta(\Omega, z)=\frac{E_{0}}{2 \cdot \sqrt{\pi} \gamma} E(w) \cdot \exp \left[-\frac{(w-\Omega)^{2}}{4 \gamma}\right] \cdot \exp [j \emptyset(w)]
$$

As already mentioned, $\tau_{\text {wavelet }}$ is large enough to ensure that analyzing function has only non negligible values over a spectral range lying in the neighbourhood of $\Omega$ in Fig.3. Under these circumstances, we have

$$
\varnothing(w)=\varnothing(\Omega)+(w-\Omega) \frac{d \emptyset}{d w}\left|+\frac{1}{2 !}(w-\Omega)^{2} \frac{d^{2} \emptyset}{d w^{2}}\right|_{w=\Omega}+\cdots+\left.\frac{1}{n !}(w-\Omega)^{n} \frac{d^{n} \emptyset}{d w^{n}}\right|_{w=\Omega}+\theta(w)_{\cdot w=\Omega}
$$

Neglecting the higher terms in eq.16:

$$
\emptyset(w)=\varnothing(\Omega)+\left.(w-\Omega) \frac{d \emptyset}{d w}\right|_{w=\Omega}+\left.\frac{1}{2 !}(w-\Omega)^{2} \frac{d^{2} \emptyset}{d w^{2}}\right|_{w=\Omega}+\theta(w) .
$$




$$
\begin{gathered}
\theta(\Omega, z)=\frac{E_{0}}{2 \cdot \sqrt{\pi} \gamma} \sqrt{\frac{\pi}{\Gamma}} \exp \left[-\frac{\left(w-w_{0}\right)^{2}}{4 \Gamma}\right] \cdot \exp \left[-\frac{(w-\Omega)^{2}}{4 \gamma}\right] \cdot \exp \left[j \emptyset^{(0)}+j(w-\Omega) \emptyset^{(1)}+\right. \\
\left.\frac{1}{2} j(w-\Omega)^{2} \cdot \emptyset^{(2)}\right] \\
\theta(t, z)=\frac{1}{2 \pi} \int_{-\infty}^{+\infty} \theta(\Omega, z) \cdot \exp (j w t) d w
\end{gathered}
$$

We calculates the temporal electric field associated with the wavelet $\theta(\Omega, z)$.

$$
\begin{aligned}
& \theta(t, z)=\frac{1}{2 \pi} \frac{E_{0}}{2 \cdot \sqrt{\pi} \gamma} \sqrt{\frac{\pi}{\Gamma}} e^{\left[-\frac{\left(\Omega-w_{0}\right)^{2}}{4 \Gamma}\right]} e^{\left(j \emptyset^{(0)}\right)} \times e^{-\left[\frac{1}{4 \Gamma}+\frac{1}{4 \gamma}-\frac{1}{2} j \emptyset^{(2)}\right] \Omega^{2}} \cdot e^{\left[\frac{\left(\Omega-w_{0}\right)}{2 \Gamma}-j \emptyset^{(1)}\right] \Omega} \\
& \times\left(\int_{-\infty}^{+\infty} e^{-\left[\frac{1}{4 \Gamma}+\frac{1}{4 \gamma}-\frac{1}{2} j \emptyset^{(2)}\right] w^{2}} \cdot e^{\left[\frac{1}{4 \Gamma}+\frac{1}{4 \gamma}-\frac{1}{2} j \emptyset^{(2)}\right] 2 \Omega w} \times\right. \\
& \left.e^{\left[-\frac{\left(\Omega-w_{0}\right)^{2}}{2 \Gamma}-j \emptyset^{(1)}\right]} \cdot e^{j w t}\right) d w
\end{aligned}
$$

The amplitude of the incident $\Omega$ wavelet is given from eq. 26 by

$$
\begin{aligned}
\theta(t, z) & =\frac{E_{0}}{2 \cdot \sqrt{\pi} \gamma} \sqrt{\frac{\Gamma(z)}{\Gamma}} \cdot \exp \left(j \emptyset^{(0)}\right) \exp \left(-\Gamma(z)\left[t+\frac{z}{V_{g}(\Omega)}\right]^{2}\right) \\
& \times \exp \left(-\frac{\left(\Omega-w_{0}\right)^{2}}{4 \Gamma}\left[1-\frac{\Gamma(z)}{\Gamma}\right]\right) \cdot \exp \left[j\left(1-\frac{\Gamma(z)}{\Gamma}\right) \Omega+\frac{\Gamma(z)}{\Gamma} w_{0}\right]\left(t+\frac{z}{V_{g}(\Omega)}\right) .
\end{aligned}
$$

This wavelet is characterized by a Gaussian envelope. This decomposition is valid only for the values of $\Delta w$ much larger than $\delta w(\Delta w \gg \delta w)$.

The delay of group of the wavelet $\left[t+\frac{z}{V_{g}(\Omega)}\right]$ is characterized by a Gaussian envelope which is the temporal width.

The delay of group of the wavelet is inversely proportional to the velocity of group its envelope propagates without deformation (Khelladi, 2008).

\subsection{Simulations}

\section{Parameters of the Simulations}

Pulse initial: $\quad \Delta \tau_{0}=10 \mathrm{fs}$

Wavelength: $\quad \lambda=800 \mathrm{~nm}$

Pulse of the wavelet: $\Delta \tau_{\text {wavelet }}=1000 \mathrm{fs}$

Longer of the medium: $z=20 \mathrm{~cm}$

Fig.21.(a) Initial pulse, (c) pulse after propagation of the $20 \mathrm{~cm}$ in litharge medium,

(e) Contour of the wavelet, $(\mathrm{g})$ the wavelet Representation,

(b) Initial pulse, (d) pulse after propagation of the $20 \mathrm{~cm}$ in the silica medium,

(f) Contour of the wavelet, (h) the wavelet Representation [6]. 


\section{Conclusion}

Finally, we have demonstrated here the possible decomposition of an ultrashort pulse into an infinite number of longer Fourier transform limited wavelets which propagate without any deformation through a dispersive medium. After propagation through the medium, the pulse may be visualized in a three-dimensional representation by the locus of the wavelet maxima. This representation permits the evaluation of the broadening suffered by the pulse. For a transparent medium, the propagation of the $\Omega$ wavelet is described by the convolution of the incident $\Omega$ wavelet with a $\theta(\Omega)$ distribution centered at the group delay relative to $\Omega$.

The application to absorbing media is relatively straightforward and will be presented in a further publication, as well as a generalization to nonlinear media. The time-frequency representation is peculiarly suitable to the latter case for which the refractive index is phenomenological time dependent. Although this technique represents a vast improvement in our ability to describe such pulses, they require additional effort, both in the apparatus and in the extraction of the pulse intensity and phase from the experimental trace.
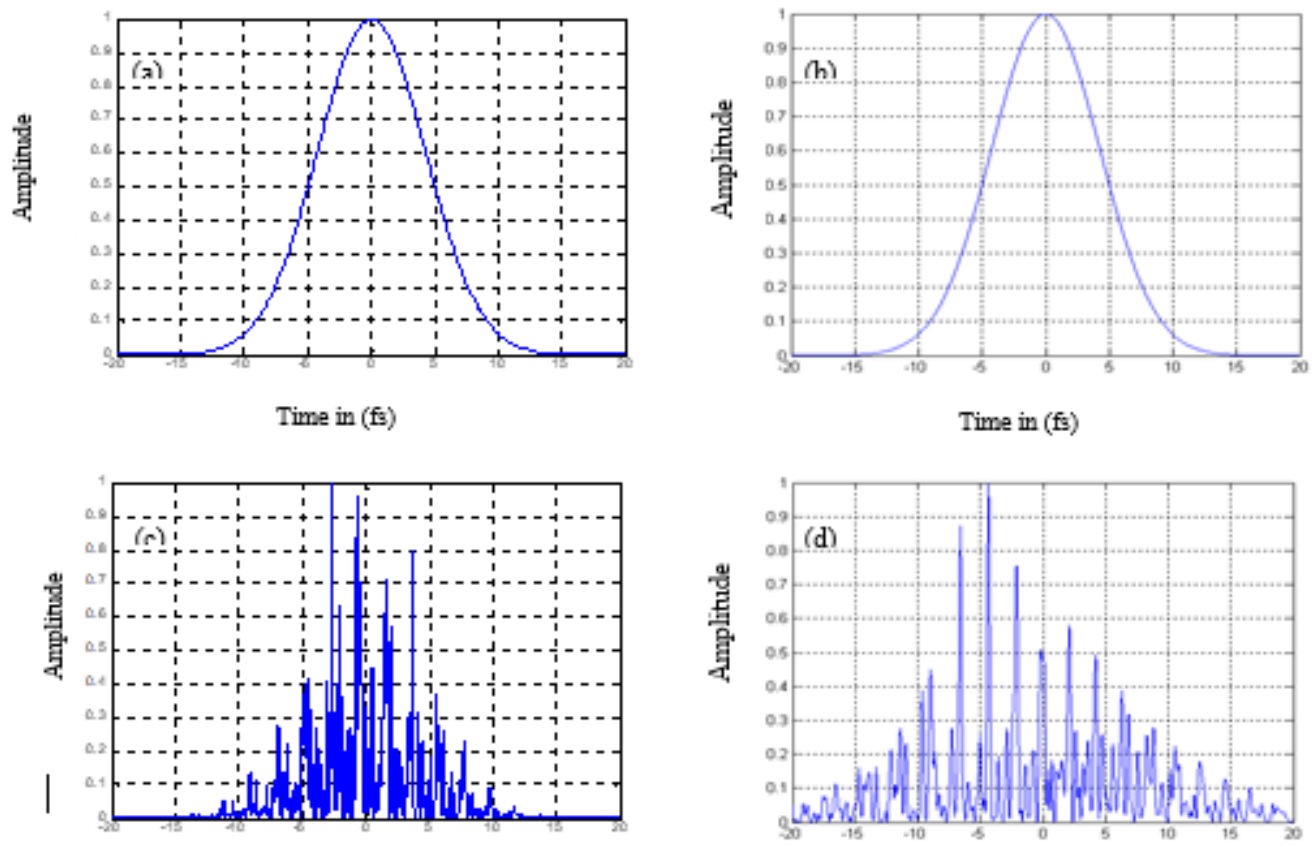

Time in (fs)

Time in (fs)
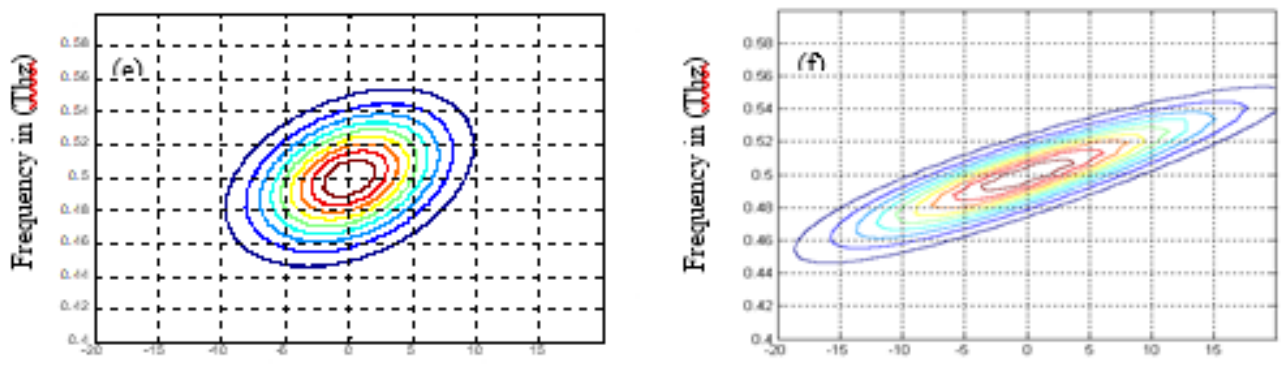

Time in (fs)

Time in (fs) 

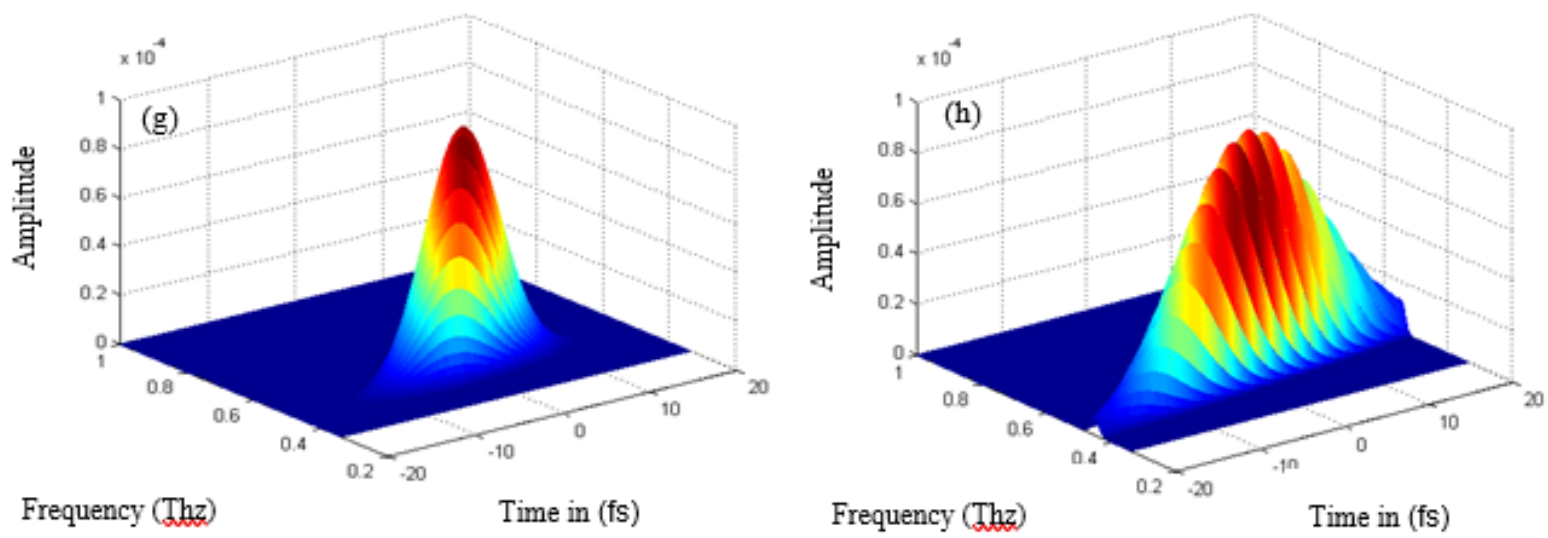

\section{References}

[1] Florenţa,A,C. "Dynamics of Ultra-short Laser Pulse Interaction with Solids at the Origin of Nanoscale Surface Modification" geboren am 16.11.1971 in Berca, Rumänien Cottbus (Florenta, 2006)

[2] Rulliere, C. Femtosecond laser pulses: principles and Experiments, Springer, (Rulliere, 1998)

[3] Fuchss, U. Zeintner, U.D., and Tunnermenn A.Ultrashort pulse propagation in complex optical systems, Opt. Express 13, 3852-3861. (Fuchss, et al. 2005).

[4] Holzwarth. "Measuring the Frequency of Light using Femtosecond Laser Pulses" aus Stuttgart den 21. December (Holzwarth ,2000)

[5] Jong Kook K. Investigation of high nonlinearity glass fibers for potential applications in ultrafast nonlinear fiber devices, Dissertation submitted to the Faculty of the Virginia (Jong Kook, 2005)

[6] Khelladi,M. et al. Time-Frequency Decomposition of an Ultrashort Pulse:Wavelet Decomposition, Radioengineering, April Vol.17, (Khelladi et al, 2008) pp.1210-2512, ISSN

[7] Meyer,Y. Jaffort, S., Riol,O. Wavelet analysis, edition Française de scientifique American (Meyer et al,1987)

[8] Khelladi,M. Ultra-short lasers pulses Characterizations: Wavelet decomposition, University of Tlemcen. Algeria, (Khellad, 2005)

*Corresponding author.

E-mail address: mo.khelladi@gmail.com 